\title{
A regulação no setor público de saúde no Brasil: os (des) caminhos da assistência médico-hospitalar
}

\author{
Regulation in the public health sector in Brazil: \\ the (mis) direction of hospital care provision
}

\author{
Sidney Feitoza Farias ${ }^{1}$ \\ Garibaldi Dantas Gurgel Jr. ${ }^{1}$ \\ André M onteiro Costa ${ }^{1}$ \\ Rodrigo de Lino Brito ${ }^{1}$ \\ Roberta Rebelo Buarque ${ }^{1}$
}

${ }^{1} \mathrm{Núcleo} \mathrm{deEstudosem}$ SaúdeColetiva, Centro de Pesquisas Aggeu

M agalhães. Av. Professor

M oraes Rego $\mathrm{s} / \mathrm{n}$, Cidade

Universitária. 50670-420

Recife PE.

sidney@cpqam.fiocruz.br
Abstract $\mathrm{H}$ ealth care regulation is a fundamental action in order to achieve effectivenessin health system. This articleanalyses SU S [ U nified $\mathrm{H}$ ealth System] main regulatory framework, in relation to historical patterns of health service delivery, observed between 1996 and 2006. Despiteall those political and organizational changes required by the Brazilian health sector reform process to implement SUS, which progressed, evidences and empirical data suggest that the agreed regulatory frameworksare weak and fragileand they did not change historical patterns and the main characteristics of SU S health care. This article suggests one possible explanatory reason for this behaviour, that needs to be empirically analysed: interest groups acting inside the Brazilian health system, on behalf of social insurance health carenetwork, could capture the regulatory process, taking public resources to this network which maintain the same pattern of heath care services for decades, jeopardizing SUS implementation and the relevant health service delivery for the Brazilian society.

Key words Health policy, Regulation in the public health sector, $\mathrm{H}$ ealth service delivery
Resumo A regulação é uma função pública imprescindível para garantir maior efetividade às ações desenvol vidas pelos sistemas de saúde. N esse estudo, são analisados os principais instrumentos de regulação da assistência à saúde criada pelo Sistema Ú nico de Saúde (SU S), vis-à-vis a evolução histórica da prestação de serviços hospitalares entre 1996 e 2006. A pesar das mudanças políticas e organizacionais exigidas pelo processo de reforma sanitária na implementação do SUS, e dos avanços detectados, evidências e dados empíricos sugerem incipiência e fragilidade dos mecanismos de regulação pactuados, que não foram capazes de mudar os padrões históricos eas características gerais da assistência hospitalar no SU S. 0 artigo apresenta uma possibilidade explicativa preliminar para a ação de grupos de interesse que teriam partici pado ativamente do processo de re gulação, canalizando recursos públicos para os segmentos dos prestadores privados e filantrópicos de serviços assistenciais, que atuam no setor saúde brasileiro há décadas e se perpetuaram no SU S, distorcen do o processo de implementação do sistema e a prestação de serviços de alta relevância para a sociedade brasileira.

Palavras-chave Análise de políticas, Regulação no setor público de saúde, Serviços hospitalares 


\section{Introdução}

A regulação é uma das funções essenciais do Estado em qualquer estágio de desenvolvimento, responsável, por definição, dentre outras questões, por regras e padrões de contratos necessários ao provimento e à prestação de serviços de alta relevância e qualidade para a sociedade. Do ponto de vista teórico, a regulação de qualquer sistema complexo visa manter suas di versas funções operativas dentro de balizas ou limites predeterminados para garantir que o sistema, no seu todo, atinja seus objetivos essenciais ${ }^{1}$.

De acordo com pressupostos neoclássicos da microeconomia, baseados na lei da oferta e demanda, a regulação dos agentes econômicos seria assegurada pela livre competição que define meIhor a quantidade ótima para produção em uma dada sociedade/mercado. N este sentido, o preço é determinado quando se alcança um perfeito equilíbrio entre oferta ea demanda. Segundo essa corrente científica, considerada mainstream, as forças de mercado devem atuar em perfeitas condições de competição; caso contrário, ocorrem as falhas de mercado. A presença eintensidade dessas falhas justificam, em maior ou menor grau, a intervenção do Estado na economia. N esta concepção, o equilíbrio é atingido quando os produtores maximizam seu lucro e os consumidores maximizam a sua utilidade, ou seja, o mercado al cança o máximo de utilidade possível, dados os recursos disponíveis na sociedade².

Segundo $\mathrm{Fiani}^{3}$, o processo de regulação é complexo, dependendo do contexto institucional específico e dos agentes que esse contexto admite como participantes do processo. Com um recorte político-institucional, essa corrente da economia estuda a regulação a partir da atuação de grupos de interesse. Os principais modelos que fundamentam essa vertente apresentam conclusões díspares quanto aos resultados. Observamse tanto processos de captura, nos casos em quea regulação beneficia a indústria regulada - denominado resultado forte - quanto estudos que apontam que a regulação surgiria como resposta à ação de diversos e complexos grupos de interesse, e não apenas dos produtores - denominado resultado fraco. N essa abordagem, ideologia, princípios, valores einteresses políticostêm papel importante na análise do processo regulatório.

Um olhar, mesmo superficial, aponta que, pela complexidade que se reveste o caso da saúde, nenhuma das condições de perfeita competição do ponto de vista microeconômico estaria presente e, assim, justifica-se uma ação mais in- tensa do Estado², que tem como objetivo, em tese, a construção de uma sociedade saudável. No setor saúde, o mercado é imperfeito, restringindo os cuidados de saúde aos consumidores com capacidade para pagar e não a todos que dele necessitam. A função reguladora do Estado é fundamental para harmonizar e articular oferta e procura em situações tão particulares, não estando em causa o cumprimento das mesmas regras da economia geral.

Além desses aspectos, já discutidos em textos acadêmicos clássicos no campo da welfare economics, a assimetria de informações é outra característica do setor saúde, facilmente evidenciada, que oferece substrato empírico à teoria do agente-principal e fundamenta a montagem das agências reguladoras independentes e a discussão dos marcos regulatórios para a coordenação dos agentes econômicos públicos e privados na área de saúde.

Na maioria dos países ocidentais democráticos, a assistência à saúde, mais do que um direi to constitucional, éconsiderada um direito da cidadania, observando-se o conceito de seguridade social. Isto implica uma responsabilidade crescente do Estado e das instituições democráticas numa soci edade plural, assentada em pactos políticos para a construção de uma agen da de direitos que visam à inclusão social e à redução de desigualdades, dentre eles os caminhos para garantir o acesso universal à saúde, considerada um bem público. $N$ estes sistemas, o debate sobre o papel dos segmentos público e privado na assistência à saúde é conduzido em função da ne cessidade de pactos em diversos níveis, objetivando viabilidade, sustentabilidade eo contorno de problemas inerentes à gestão pública eà complexidade do setor saúde. Dentre as questões estruturais e dinâmicas disfuncionais, observadas em qualquer sistema de saúde, evidenciam-se os desencontros e/ou desequilíbrios entre a oferta do sistema, a demanda e a escolha dos consumidores $^{4}$, que impõe necessidades de racionalização da assistência à saúde que o livre mercado não é capaz de assegurar.

Por outro lado, padrões adequados de acesso, resolutividade, excelência na qualidade da prestação do serviço e acolhimento formam um verdadeiro calcanhar de Aquiles na montagem desses sistemas nacionais de saúde, sobretudo naqueles quetêm forte presença estatal no provimento da assistência. Sob o prisma da equidade na assistência a saúde, pode-se inferir que a re gulação é imprescindível e sua finalidade é assegurar que se atinjam os grandes objetivos sociais 
do sistema de saúde, equilibrando as numerosas falhas de mercado e/ou fal has do governo que caracterizam esse setor. Afinal, nas sociedades que alcançaram alto nível de organização dos seus sistemas nacionais, a saúde não é um bem privado de consumo ou de permuta, mas tem como característica estrutural preços elevados e pressão de custos crescentes.

M agalhães Júnior ${ }^{5}$ afirma que regulação não ésinônimo de gestão, mas um de seus elementos constituintes e uma importante ferramenta para sua operacionalização, tratando a regulação no SUS de duas maneiras, ambas inter-relacionadas à gestão global do sistema: a macrorregulação e a microrregulação. A primeira significaria uma articulação e prática de mecanismos estratégicos de gestão que podem se estabelecer em bases sociais públicas - como as defendidas no processo da Reforma Sanitária Brasileira - em bases políticas menores, em bases corporativas ou tecnocráticas ou ainda sintonizadas com os interesses privados do mercado; a segunda refere-se a um olhar diretamente para a assistência, com recorte operacional e instrumental.

A atuação de diversos grupos de interesses, envolven do diretamente a contratualização, produção e o controle do desempenho dos agentes que atuam no sistema, torna a regulação uma área crítica, permeada de conflitos. Ressalvando-se a necessidade de aprofundar aspectos epistemológicos e conceituais fundamentais ainda frágeis encontrados na literatura brasileira sobre o Sistema Único de Saúde (SUS), abordaremos questões relacionadas à "macrorregulação" da assistência médico-hospitalar dirigida aos prestadores de serviços de saúde públicos e privados contratados pelo SUS.

Ao longo desses vinte anos de existência, 0 SUS apresenta números imponentes, dignos do maior projeto público de inclusão social no Brasil $^{6}$. Conforme dados do Ministério da Saúde (MS) são realizados, em média, por ano, onze milhões de internações, 1,4 bilhão de procedimentos ambulatoriais, 44 milhões de consultas especializadas e 250 milhões de consultas básicas por uma enorme rede, composta de mais de 5,8 mil hospitais, sessenta mil unidades ambulatoriais e quinhentos mil leitos?.

A despeito dessa magnitude, o SU S enfrenta proporcional mentegigantescas dificuldades, pela complexidade do desafio representado pela sua implementação, tais como problemas crônicos no financiamento, incipiente regulação, precariedade das relações de trabal ho efalhas no modelo de gestão descentralizada. Como consequên- cia, há muitos hospitais mal distribuídos pelo território efaltam leitos, qualidadee eficiência $a^{6,8}$.

Politicamente, os processos de regulação são extremamente disputados e submetidos a várias pressões e tensões. No Brasil, o comportamento dos atores sociais em situação ou a ação dos grupos de interesse são institucionalizados em verdadeiras arenas de conflito, dentro do aparelho de Estado, via Ministério da Saúde, Comissões Intergestores, Consel hos e Conferências de Saúde que estabelecem os canais de participação dos principais atores sociais na política de saúde. Isto confere, a nível setorial, a necessidade de pactos para efetivamente modular a política e equacionar as demandas sociais na busca de satisfazer as necessidades e representações da população ${ }^{9}$.

No processo de institucionalização do SUS, a introdução de ações de natureza regulatória vem sendo caracterizada pela edição, no âmbito jurídico-institucional, das chamadas Leis Orgânicas da Saúde (leis nº 8.080 e n 8.142 de 1990) e dos pactos administrativos, via N ormas O peracionais Básicas (NOB/SUS), que representam instrumentos de regulação para o processo de descentralização. Foram expedidas N OB em 1991, 1992, 1993 e 1996 e também foram elaboradas as Normas O peracionais da Assistência à Saúde (N OAS 01/2001 e 01/2002). M ais recentemente, estamos sob a vigência do Pacto pela Saúde 2006?.

A pesar dos avanços conceituais detectados e das profundas transformações na responsabilidade das três esferas de governo, decorridos do processo de descentralização, a regulação ainda tem se caracterizado como eminentemente cartorial. Evidências e dados empíricos sugerem que não foram modificados suficientemente aspectos importantes da assistência, tais como forma de definição e seleção das prioridades, ordenamento da distribuição e expansão da oferta de serviços e inadequação entre demanda e oferta, que persistem como heranças do modelo assistencial da medicina previdenciária regulado pelo Instituto N acional de Assistência M édica da Pre vidência Social (INAM PS), anterior ao SUS.

A proposta original do SUS era universalizar, prioritariamente, ampliando a cobertura pública do conjunto da assistência para toda população; entretanto, os dados do Sistema de Informações Hospitalares do SUS (SIH/SUS ${ }^{7}$ ) mostram que isso de fato não ocorreu nacionalmente. A Pesquisa de Assistência M édico-Sanitária (AM S) ${ }^{10}$ demonstra que ocorreram avanços na oferta dos serviços de saúde. Contudo, observase no SUS uma enorme presença de serviços de assistência médico-hospitalar do setor privado 
conveniado/contratado complementar, ausência ou debilidade dos contratos formais ${ }^{11}$ e incipiência efragilidade dos mecanismos de regulação da assistência à saúde no Brasil.

N este artigo, examinaremos a regulação na assistência médico-hospitalar, os marcos da descentralização e a introdução de medidas explícitas de regulação no período entre1996-2006. Para traçar o caminho percorrido pelos serviços de saúde, utilizaremos a base de dados do SIH/SUS, importanteferramenta deabrangência nacional ${ }^{12}$.

\section{Localizando conceitual mente a regulação}

Dentre outras razões, que alicerçam o poder coercitivo do Estado e o seu papel regulador sobre o mercado, encontra-se a necessidade da garantia de padrões aceitáveis e previsíveis para a prestação de serviços à sociedade, que livremente 0 mercado per si não é capaz de garantir. Estas atividades vêm sendo, ao longo do último século, marcadamente exercidas, nos países liberais democráticos ocidentais, através de um conjunto de leis que normatizam a relação Estado-mercado-sociedade.

Entretanto, alguns el ementos importantes diferenciam ainda mais a regulação pública da regulamentação ou normatização geral, que o Estado já exerce constitucionalmente, sobretudo após o processo de reformas estruturais observadas a partir da década de noventa. Para Selznike, citado por Walsh $\mathrm{e}^{13}$, mais especificamente, a regulação se refere basicamente à ação de uma agência pública sobre serviços de val or para a coletividade. Essa ação pressupõe uma autoridade pública formalmente constituída, que centraliza as ações de regulação, atuando por fora das relações comerciais egovernamentais, agindo em função do interessepúblico. Esse conceito apontapara a autonomia que as atuais agências reguladoras têm em relação ao poder executivo e as pressões dos ciclos de disputa político-eleitoral. Ela também se diferencia da simples regulamentação imposta tradicionalmente pela aplicação de leis gerais, que qualquer cidadão pode acionar via órgãos do Poder Judiciário. A regulação pode ainda ser melhor entendida, na atualidade, pelo poder queum ente público independentecom mandato oficial detém para formatar enormatizar o funcionamento de organizações que prestam serviços específicos à sociedade, quer seja em caráter público e/ou privado.

N este campo particular do conhecimento, váriosmodelos deregulação pública vêm sendo cons- truídos. Um dos mais didáticos se baseia em ações escalonadas numa estrutura piramidal, onde na base está a elaboração de acordos operacionais; em seguida, no nível intermediário, dá-sea definição de regras e padrões contratualizáveis em quantidade e qualidade dos serviços até punições aos prestadores que aparecem no topo do modelo, que poderia se chamar de marco regulatório ${ }^{14}$. Alguns modelos de regulação foram desenvolvidos com ênfase em ações de natureza punitiva; outros apresentam um processo de regulação compreensiva baseado em ações educativas. Ainda há os que unem as duas formas de abordagem, sendo classificados como de regulação responsiva.

0 modelo regulatório adotado depende da situação a ser regulada, da concepção do agente regulador edo ambienteorganizacional dos prestadores de serviço. A regulação por agência, atual mente utilizada, situa-se entre o modelo de autorregulação pelo mercado, tipo laissez-faire, sujeito às suas falhas, e o modelo de regulação burocrática do tipo cadeia de comando econtroleestatal, da mesma forma suscetível à captura e/ ou insulamento burocrático. As estratégias de regulação de cada seguimento definem e determinam a existência de tipos predominantes de regulação, tais como regulação do tipo estatal normativa e/ou escal onada, tripartitismo, autorregulação contratada e autorregulação com vários órgãos públicos envolvidos no processo re gulatório em diversos níveis.

Sinteticamente, do ponto de vista dos objetivos funcionais, a regulação está assentada em dois grandes pilares: quantidade adequada de ações e serviços acessíveis e resolutivos aos cidadãos e a boa qualidade dos padrões dos serviços ofertados. Estes objetivos definem a necessidadedeações no plano estratégico, tático-operacional einstrumental, caracterizando-se como função importante na relação entre o Estado, o setor público de forma ampliada, a sociedade e os mercados.

0 processo inflacionário que ocorre nos sistemas ao redor do mundo tem tido grandes re percussões na gestão dos sistemas universais e no processo de trabalho das organizações do setor saúde. Segundo Almeida ${ }^{15}$, esse fenômeno abre caminho para inúmeras propostas de re formas racionalizadoras e inovações gerenciais em todos os sistemas de saúde nas últimas décadas. A expansão das despesas com saúde é, por razões diversas, objeto de preocupação em todos os países. N estes sistemas, as relações estabelecidas com os prestadores de serviços de saúde podem ser um dos principais motivos da escalada dos custos ou mesmo seu principal indutor. 
Por outro lado, maior efetividade e eficiência na prestação de serviços parecem ser obtidas com a regulação, como uma ação estratégica, sobre prestadores de serviços e provedores da área de saúde ${ }^{16}$, sobretudo na seleção de prioridades para a oferta de serviços. N este contexto, observa-se uma nítida ten dênciainternacional de contenção da liberdade de prática dos profissionais, no sentido de estabelecer um padrão de contratualização e racionalidade no uso dos meios de diagnóstico etratamento. De acordo com Harrison e Smith $^{17}$, estamos diante de uma nova burocracia que desafia as relações estabelecidas pelas burocracias profissionais, sobretudo quanto a sua autonomia no processo de trabalho.

Ações reguladoras já estão presentes nos modelos de manage care usados no gerenciamento do setor privado e nos contratos de gestão para 0 setor público, com forte rebatimento sobre as organizações que prestam assistência eno exercício das profissões liberais, suscitando a necessidade de novos pactos. Apesar de desafiar o poder das grandes corporações e das burocracias profissionais, estruturalmentetem-seobservado queéprecisamentea falta desses pactosquetem o potencial de anular medidas de controle e determinar modalidades de cuidados econsumo de serviços ${ }^{18}$.

Os limites financeiros de sistemas de saúde aumentam a necessidade de regulação da oferta, demanda e acesso aos serviços. O SUS não fogeà regra e necessita ação regulatória apropriada a suas características. Questões como o descontrole do acesso espontâneo pelos usuários e a liberdade na definição da oferta de serviço pelos provedores e profissionais de saúde podem levar à superprodução desnecessária, mesmo por zelo ou conduta ético-profissional apropriada, como bem enfatizado por Harrison e M oran ${ }^{19}$, observando esse fenômeno em vários países.

Os (des) caminhos da regulação da assistência à saúde no Brasil

No Brasil, os problemas decorrentes da crise do financiamento do setor público durante os anos oitenta, com o consequente sucateamento da rede pública de saúde, aliados aos incentivos governamentais eà renúncia fiscal via ben efícios agregados ao salário do trabalhador, contribuíram para o expressivo aumento da clientela beneficiária de planos privados de saúde, que de certa forma esvaziaram os órgãos de controle social e consequentemente sua capacidade de regulação da política de saúde no SUS. Num contexto am- plo, tanto Favaret e Oliveira ${ }^{20}$ como $^{\mathrm{M}}$ endes $^{8}$ defendem que há uma relação entre o desfinanciamento do setor público de saúde, pelas modificações no processo de financiamento setorial que determinaram as flutuações e a turbulência dos anos noventa ${ }^{21}$, e a queda da qualidade dos serviços prestados no SUS, com o crescimento do setor supletivo brasileiro, como contornos da universalização excludente ${ }^{20}$; portanto, a frágil capacidade de regulação dos consel hos de saúde ajuda a desviar o caminho da implementação do SUS de suas bases constitucionais.

É inegável o avanço conquistado pelo processo de descentralização das ações eserviços de saúde para a assistência médico-hospitalar no SUS. Entretanto, al gumas questões são desafios à reestruturação do sistema de saúde, tais como a perda de escala decorrente da municipalização autárquica e da persistência de serviços hospitalares de pequeno porte com menos de cinquenta leitos quetêm baixacomplexidadeeresolutividadequestionável. Esses hospitais foram credenciados há décadas e submetidos à lógica da produção que determina o número de autorizações de internações hospitalares (AIH) para cobrir seus custos, independente da sua eficiência e qualidade dos serviços prestados. A ausência de uma política de regulação efetivano SU Sexplicita problemas como presença de leitos e falta de vagas para diversas modal idades de tratamento, fulcro da necessidade de regulação do sistema no quese refereà adequação da oferta à demanda e, consequentemente, à questão da resolutividade e racionalidade, distorcendo a prestação da assistência a saúde.

Outro fenômeno importante observado no processo de implementação do SUS é a enorme participação do setor privado complementar. Observando os dados de internação do SIH/SUS no período de 1996 a 2006, verificamos que 0 valor total e médio das internações pagas aos hospitais filantrópicos apresenta uma tendência de crescimento, sendo superior à média dos hospitais públicos (Gráficos 1 e 2).

Do total de 11.338 .039 internações ocorridas em 2006 (Tabela 1), o setor privado/conveniado foi responsável pela realização de $56,6 \%$, repre sentando um montante de $R \$ 6.998 .043 .548,10$, ou seja, al go em torno de $60,4 \%$ dos gastos com internações em 2006.

Estes dados demonstram um crescimento expressivo das internações em hospitais públicos, porém revelam a falsa complementaridade do setor privado/conveniado, que nascee permanece superando os hospitais públicos na rede de assistência à saúde do SUS. 


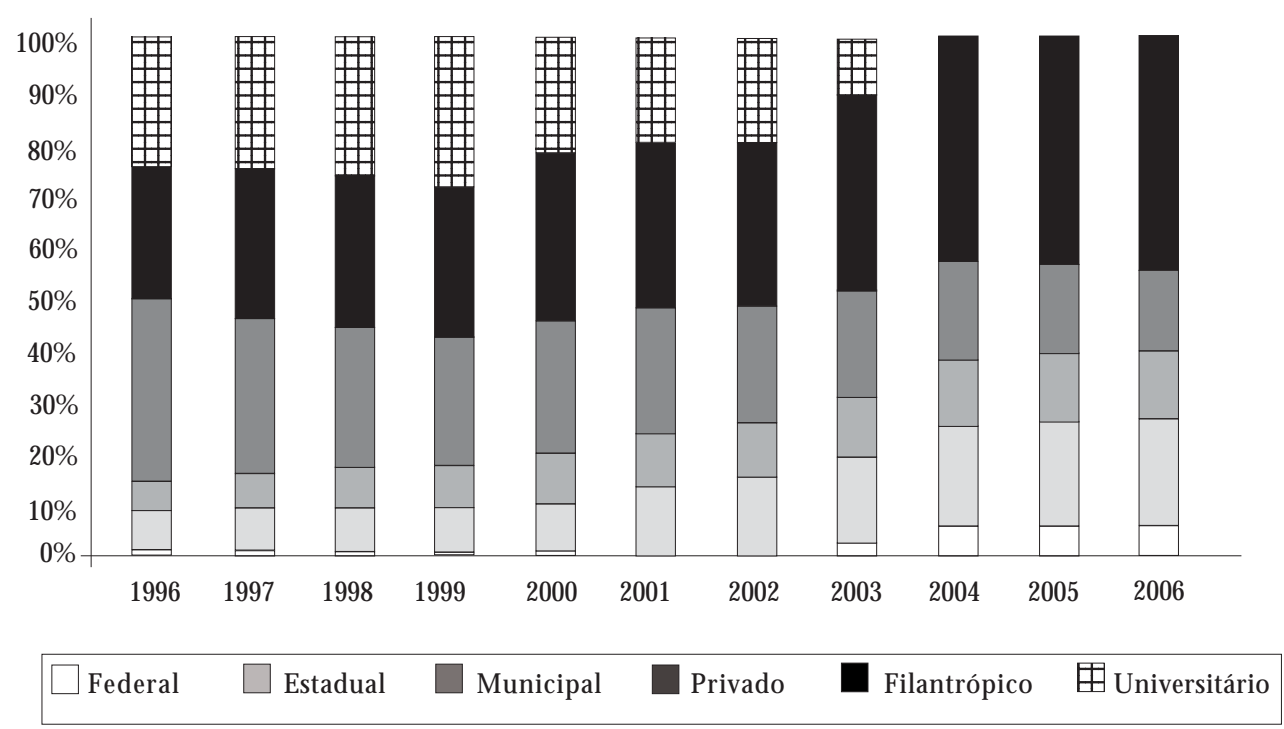

Gráfico 1. Evolução percentual das internações hospitalares por valor total segundo natureza do prestador. Brasil, 1996-2006.

Fonte: SIH/SUS.

Obs.: A partir de agosto/2003, as unidades universitárias foram reclassificadas em públicas e privadas.

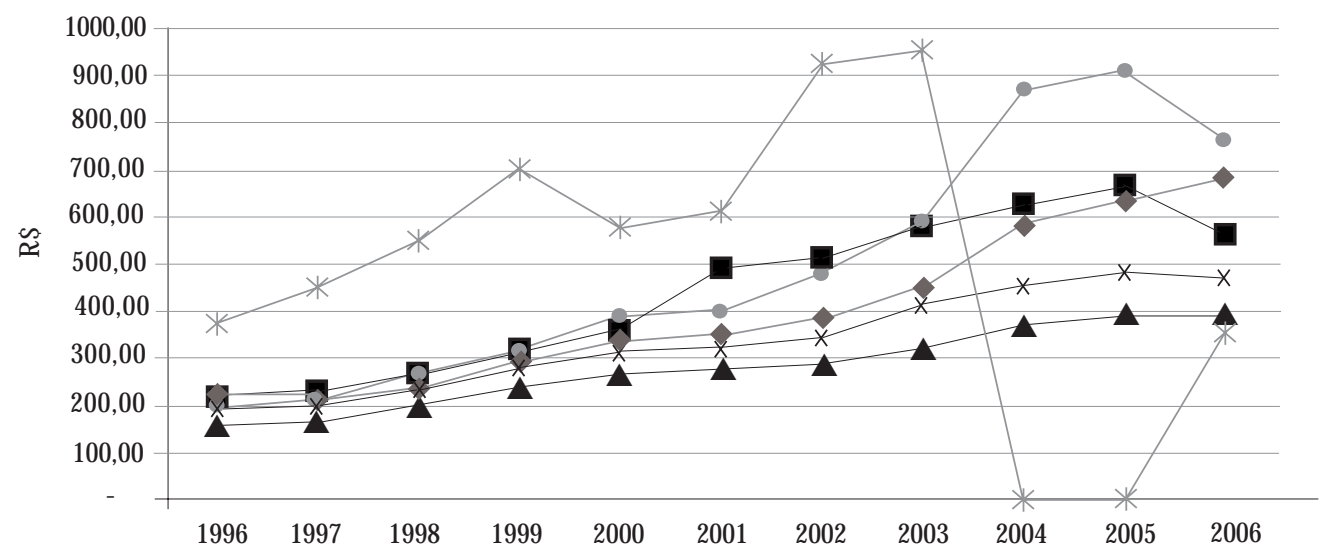
- Federal
- Estadual
- Municipal $*$ Privado
Filantrópico
* Universitário

Gráfico 1. Evolução percentual das internações hospitalares por valor total segundo natureza do prestador. Brasil, 1996-2006.

Fonte: SIH/SUS.

Obs.: A partir de agosto/2003, as unidades universitárias foram reclassificadas em públicas e privadas. 
A baixa capacidade de regular a ação desses agentes privados, no processo de reforma estrutural, é um problema prioritário na agenda dos gestores em todos os países que garantem o direito universal e permitem a participação crescente do setor privado na provisão dos serviços de assistência. O Brasil apresenta inúmeras contradições quando se observam os marcos regulatórios legais pactuados e a implementação do sistema.

Assim, observa-se que o número de unidades hospitalares credenciadas privadas que em 1996 representava em torno de 37\% das internações em 2006 cai para apenas 16\% (Tabela 1). Entretanto, as unidades filantrópicas isentas do recolhimento deimposto derenda, que não existiam em 1996, passam a ser responsáveis por quase $1 / 3$ do valor pago pelas internações em 2006. Outro dado relevante é que, mesmo reduzindo-se em $10 \%$ o percentual das internações, 0 setor conveniado permanece com a mesma parcela dos $60 \%$ dos recursos financeiros das internações hospitalares do SUS, ou seja, os dados indicam que estas unidades são duplamente favorecidas: aumento do valor médio das internações e ben efícios fiscais.

Os dados de internações parecem corroborar com questionamentos feitos sobre a regulação do setor conveniado. Conforme advertia Costa22, a definição nebulosa do artigo 199 sobre a participação das instituições privadas no SUS, mediante contrato de direito público ou convênio, ea preferência às entidades filantrópicas sem fins lucrativos, pode justificar este quadro atual. Segundo o autor, "no [...] manto de 'entidades sem fins lucrativos' estão muitas instituições cuja única finalidade é o lucro", particularmente no caso de algumas especialidades melhor remuneradas sem nenhum fundamento técnico.

Por outro lado, a manutenção da prestação de serviços, que apresenta forte descompasso com o poder de resolutividade e necessidades da população, simplesmente reproduzindo a série histórica de autorizações de internação hospitalar do extinto INAM PS, é uma evidência da fragilidade do processo regulatório. As evidências apontam que as faturas apresentadas pelos prestadores ao órgão previdenciário que centralizava essas funções, após o processo de municipalização, foram simplesmentetransferidas para o gestor municipal autorizar, reforçando o descaminho no SUS.

Os resultados da pesquisa da $\mathrm{AM} \mathrm{S}^{10}$, com relação à internação, mostram que o total de unidades hospitalares conveniadas que prestam atendimento ao SU Satingiu $78,9 \%$, ou seja, mais de 2/3 são de serviços "contratados". Quase 70\% das unidades privadas com internação atendem ao SUS. Apesar da Constituição de 1988 assegu-

Tabela 1. Comparativo entre quantidade e valor total das internações hospitalares no SUS segundo a natureza do prestador. Brasil, 1996 e 2006.

\begin{tabular}{|c|c|c|c|c|c|c|c|c|}
\hline \multirow[b]{3}{*}{ Natureza } & \multicolumn{4}{|c|}{ Internações hospitalares } & \multicolumn{4}{|c|}{ Valor total } \\
\hline & \multicolumn{2}{|l|}{1996} & \multicolumn{2}{|c|}{2006} & \multicolumn{2}{|c|}{1996} & \multicolumn{2}{|l|}{2006} \\
\hline & Qtd. & $\%$ & Qtd. & $\%$ & Qtd. & $\%$ & Qtd. & $\%$ \\
\hline \multicolumn{9}{|l|}{ Público } \\
\hline Federal & 156.731 & 1,31 & 431.488 & 3,81 & $30.010 .107,89$ & 0,94 & $406.957 .738,99$ & 5,82 \\
\hline Estadual & 1.139 .958 & 9,55 & 2.147 .011 & 18,94 & $251.338 .446,98$ & 7,90 & $1.436 .592 .775,00$ & 20,53 \\
\hline M unicipal & 1.172 .751 & 9,83 & 2.332 .178 & 20,57 & $189.262 .148,29$ & 5,95 & $918.818 .154,68$ & 13,13 \\
\hline Subtotal & 2.469 .440 & 20,69 & 4.910 .677 & 43,32 & $470.610 .703,16$ & 14,79 & $2.762 .368 .668,67$ & 39,48 \\
\hline \multicolumn{9}{|l|}{ Privado/Conveniado } \\
\hline Privado & 4.368 .985 & 36,61 & 1.841 .371 & 16,24 & $1.114 .316 .004,77$ & 35,01 & $1.109 .450 .917,60$ & 15,85 \\
\hline Filantrópico & 3.615 .411 & 30,30 & 1.255 .477 & 11,07 & $801.524 .970,81$ & 25,19 & $720.449 .135,01$ & 10,30 \\
\hline Fil. isento Trib. e cont. sociais & - & - & 3.316 .110 & 29,25 & - & - & $2.397 .176 .203,97$ & 34,25 \\
\hline Fil. isento IR e cont. s/lucro liq. & - & - & 961 & 0,01 & - & - & $717.108,85$ & 0,01 \\
\hline Subtotal & 7.984 .396 & 66,91 & 6.413 .919 & 56,57 & $1.915 .840 .975,58$ & 60,20 & 4.227 .793 .365 & 60,41 \\
\hline Universitário & 1.478 .818 & 12,39 & 843 & 0,01 & $795.814 .645,30$ & 25,01 & $295.933,95$ & 0,00 \\
\hline Ignorado & - & - & 12.600 & 0,11 & - & - & $7.585 .580,05$ & 0,11 \\
\hline Total & 11.932 .654 & 100 & 11.338 .039 & 100 & $3.182 .266 .324,04$ & 100 & $6.998 .043 .548,10$ & 100 \\
\hline
\end{tabular}

Fonte: SIH/SUS. 
rar e garantir aos cidadãos o direito à saúde e ao acesso universal e equânime às ações e serviços para sua promoção e recuperação, contraditoriamente, cresce progressivamente as queixas em relação a limites de acesso, à qualidade e às carências nos serviços de saúde, principal mente na média e alta complexidade, ocupadas majoritariamente pelo setor privado complementar. Isso faz crer que a flexibilidade advinda da contratação de organizações não governamentais, filantrópicas, privadas e/ou de qualquer outra natureza carece de forte componente regulador para o alcance da missão do sistema.

Apesar dos hospitais filantrópicos terem reconhecida importância e participação fundamental no SUS, padecem de crises determinadas por fatores relacionados à política de financiamento, ao perfil assistencial, à gestão e ao processo de inserção no sistema locorregional de saúde, como descrevea Portaria GM /M Sno 1.721/ 2005, que cria o Programa de Reestruturação e Contratualização dos H ospitais Filantrópicos no SU S. Entretanto, vale ressaltar que a trajetória sinalizada para a modernização dos hospitais públicos, tais como as flexibilizações nas contratações, nas compras e nas aquisições de insumos, etc., já fazem parte da realidade destas instituições e, mesmo assim, demonstra grande incapacidade na condução gerencial, necessitando por vezes do socorro governamental.

A relação entre a oferta da assistência médico-hospitalar pública e privada conveniadaé, porém, muito mais complexa. Do lado dos provedores privados, o que se observa é uma diversidade de relações de credenciamento. Um mesmo estabelecimento pode, por exemplo, ser conveniado ao SUS, credenciado a uma ou mais operadoras do setor suplementar e utilizado por indivíduos e famílias de forma privada ${ }^{23}$. Acrescente-se a isto a judicialização do acesso a procedimentos assistenciais de médio e alto custo, que aprofundam a iniquidadeeinsustentabilidade do sistema ${ }^{6}$.

A regulação da assistência à saúde no SUS: tornando mais explícitas as intenções

O M inistério da Saúde partiu de dois argumentos básicos para explicitar a necessi dade de regular a assistência à saúde no SUS. Inicialmente, problemas disfuncionais em torno da descentralização dos serviços no processo de municipalização do SUS a partir da N OB/96; posteriormente, a compra/oferta de serviços pelo gover- no, determinada, historicamente, pelo interesse dos prestadores dos serviços de saúde.

A N OAS 01/02 propôs que estados e municípios organizassem suas estruturas de controle, regulação e avaliação para garantir o acesso de seus cidadãos a todas as ações e serviços necessários para a resolução de seus problemas de saúde, otimizando recursos disponíveis e reorganizando a assistência, buscando a melhor alternativa em termos de impacto na saúde da população. Para a reorganização destas atividades, foram definidas atribuições básicas inerentes a cada nível do governo a serem contempladas no Plano de Controle, Regulação e Avaliação da Assistência prevista na NOAS.

Embora $M$ endes ${ }^{8}$ discuta que o principal problema da N OAS SU S 01/02 consistiu no seu foco, dirigido para a gestão da oferta e, portanto, re produzindo o erro de concepção das NOB anteriores, analisando em profundidade o texto, com olhar para a regulação explícita inserida na norma, verifica-se que apenas essa argumentação não é suficiente para compreender a manutenção dos problemas no SUS. A NOAS está fundamentada na necessidade de reconfigurar o sistema de base municipal para uma nova base locorregional e isto enfrenta forteresistência dianteda concepção municipalista prevalente, sobretudo quando é proposta a retomada do papel de condução da organização da assistência pelas secretarias estaduais no processo de regionalização. A construção de um sistema funcional impõe novas relações e certamente tem consequências para a provisão de serviços de saúde, ainda fortemente baseados no extinto mercado previdenciário. Esse é o verdadeiro problema que se arrasta há décadas no Brasil equefez a N OAS eseu processo confuso de regulação algo de difícil condução.

A NOAS poderia de fato atacar os problemas advindos da frágil regulação da assi stência à saúde e consequentemente da oferta e distribuição de serviços que têm por base a persistência das relações do mercado previdenciário no SUS. Pois a oferta de serviços no Brasil se baseia na série histórica de procedimentos realizados e contratados pela previdência pública, com forte interferência política, na qual a prestação de serviços foi definida pelos ofertantes dentro da lógica do mercado cativo, criado nas décadas anteriores à implementação do SUS. No contexto da medicina previdenciária, a prioridade era dada pelo mercado para a realização de procedimentos mais rentáveis, em serviços nos quais existia, logicamente, maior número de clientes consumidores com vínculos formais de trabalho. 
A assistência médica prestada no período anterior ao SU S era garantida aos segurados contribuintes do IN AM PS pela compra de serviços privados contratados e credenciados pagos por procedimentos realizados. Os dados eram contabilizados em sistemas de informação hospitalar e ambulatorial criados inicialmente para fins de apuração dos custos e pagamento de prestadores deserviço. 0 modelo de regulação centrava-seno controle dos gastos, apoiado num burocrático conjunto de regras efluxos, que passou a direcionar o tipo de assistência prestada pela rede credenciada para uma lógica de produção de atos isolados e de maior custo. 0 padrão de relação mercantil produtivista caracterizou a assistência no Brasil para aquela população segurada.

Esta lógica foi transferida para o SUS e inclusiveestendida a sua rede própria estatal, que passou a funcionar com base nesses mesmos princípios com a NOB/91. Toda a rede passou a se comportar da mesma forma que as organizações da rede privada credenciada, como se estivesse na condição de prestadores de serviços, re munerados pela tabela de procedimentos do INAM PS. 0 financiamento do sistema seguiu a lógica de produção de serviços, catalogados a partir de então no Sistema de Informação Ambulatorial (SIA) e H ospitalar ( $\mathrm{SIH}$ ), baseados na lista de procedimentos médicos do tipo Disease Related Groups (DRG). Esses procedimentos racionalizadores, aplicados aos prestadores privados contratados pelo sistema previdenciário, buscavam controlar principalmente a produção e os gastos na assistência médica aos segurados e foram adotados pelos gestores do SUS desde 1990, havendo poucas mudanças desde então nesse procedimento padrão dentro do processo demunicipalização.

O setor credenciado, tanto no IN AM PS quanto no SUS, atua para canalizar recursos para essa redeindependentedanecessidadee demanda real, ou seja, a oferta cristalizada passou a determinar a demanda. Esta realidade gera inúmeras distorções, como o excesso de oferta, alta concentração de serviços e superposição de prestadores e ações, em que notadamente já existia mercado consumidor com maior potencial em cidades maiores e nas regiões mais desenvolvidas. Também cria gargalos estruturais para algumas necessidades assistenciais importantes mal remuneradas ou que apresentam baixa rentabilidade, resultando em filas elistas de espera inaceitáveis.

Apesar das mudanças políticas exigidas pelo processo de reforma sanitária, os mecanismos de regulação pactuados não foram capazes de mu- dar os padrões e as características gerais da assistência no SUS. A série histórica da oferta deserviços ea lógica de controlee avaliação quantitativos estabelecidos pela assistência previdenciária do extinto INAM PS foram reproduzidas, mesmo diante de profundas mudanças no arcabouço jurídico-legal ealterações incrementais no processo normativo. A NOB 96 estabeleceu tetos financeiros globais para os municípios e passou a definir novos papéis aos entes subnacionais, sobretudo com destinação de recursos para atividades não assistenciais, tais como ações de vigilância; porém, na prestação de serviços de assistência, a lógica inampiana se perpetuou até nossos dias, mantendo a forma de pagamento por produção incorporada no teto da assistência.

A contratação dos prestadores de serviços no SUS é outro problema que tem se arrastado ao longo de sua existência, sofrendo resistências e fazendo prevalecer a informalidadeneste processo. Em 1993, o M inistério da Saúde publicou inicialmentea Portarian ${ }^{\circ} 1.286 / 93^{7}$, queestabeleceu parâmetros para contratação da rede prestadora de serviços de saúde dos gestores municipais. Em 2003, foi lançado outro documento buscando reorientar a contratação de serviços de saú$\mathrm{de}^{24}$. Com o intuito deauxiliar eacelerar este processo, o M inistério da Saúde criou em 2005 o Programa de Reestruturação dos Hospitais Filantrópicos, estabelecendo incentivo financeiro à adesão, visando à contratualização ${ }^{7}$.

A publicação da Portaria M S no 3.277/20067 institui novas diretrizes para a participação complementar dos serviços privados de assistência à saúde no âmbito do SUS. Como um pressuposto da nova política de regulação na reformulação da normatização do setor e da exi gência de contratação dos prestadores estipulada na Portaria M S no 399/2006 do Pacto de Gestão ${ }^{7}$, as diretrizes tornaram-se imperiosas.

A pesar das normas vigentes, a contração da rede prestadora de serviços apresenta falhas, é limitada e ainda não assumida plenamente pelas esferas de governo. Segundo Santos e M erhy ${ }^{25}$, Esta rede, sub-rogada pelo extinto IN AM PS aos estados, foi, também, sub-rogada aos municípios, em sua imensa maioria com os contratos vencidos, caducos, ou mesmo sem nunca ter sido assinado um convênio com o poder público, trabalhando por meio de um credenciamento, com critérios de entrada no sistema pouco definidos.

Atualmente, criam-se novas expectativas devido à publicação do Pacto de Gestão ${ }^{7}$, processo de ampla discussão que definiu as responsabilidades sanitárias dos gestores do SUS e estabele- 
ceu novas diretrizes para a gestão do sistema. M ais recentemente, com a definição da Política Nacional de Regulação em 2006, o M inistério da Saúde vem disseminando mais "um novo conceito" de regulação estatal sobre o setor saúde, "entendida como aquela em que o Estado atua sobre os rumos da produção de bens e serviços de saúde, por meio das regulamentações e das ações que asseguram o cumprimento destas"26. A pesar dos avanços legais enormativos, no mundo real, pouca coisa mudou, persistindo os problemas identificados desde a N OAS/2001, que se tornam ainda mais ambíguos devido a esse esvaziamento conceitual ebaixa capacidade operacional na efetivação dos pactos.

\section{Consideraçõesfinais}

A implantação do SUS trouxeum aumento significativo da demanda, com a inclusão de segmentos antes excluídos. Em quase vinteanos dehistória do SUS, o setor conveniado desenvolveu-se em um extraordinário contexto institucional de incipiente regulação, com incentivos fiscais e ausência de barreiras para o credenciamento. Grande parte desse setor foi incorporada automaticamente pelos compromissos assumidos e herdados dos grupos de interesses históricos que povoaram o INAM PS e a medicina previdenciária. Esse nítido descompasso não parece fruto dos efeitos não antecipados, mas sim da correlação de forças presentes na arena do setor saúde - espaço democrático - com participação plural e legitimada pel os preceitos constitucionais.

Cabe aos atores interessados em garantir o caminho da saúde para todos, inscrito na Cons- tituição Federal e nas Leis Orgânicas da Saúde, como um direito de cidadania, tracionar o movimento no rumo à outra direção, mantendo vivos os princípios e di retrizes do SUS. Para a qualificação do processo regulatório, é preciso trabalhar com a concepção ampla de regulação de todo o sistema, no qual diversas ferramentas de gestão têm o potencial de ajudar a equacionar, coordenar e melhorar a assistência.

Este cenário destacado sugere que os grupos de interesses que atuam no SUS parecem ter sido bem-sucedidos na captação de recursos públicos e na ampliação de seu espaço na rede de serviços de assistência hospitalar. Os mecanismos de regulação são frágeis, insuficientes e tímidos, como já ressaltado em estudos recentes sobre as políticas de saúde no Brasi|6,8,11,25.

Neste sentido, esse estudo ressal ta uma possibilidade teórico-explicativa que necessita ser submetida à análise empírica: a ação de grupos deinteresse seria capaz decapturar o processo de regulação do SUS, mantendo e tracionando benefícios seletivos por dentro do seu processo de implementação. 0 movimento da rede hospitalar privada no SUS mereceuma análise mais aprofundada devido à dinâmica das transformações que vêm ocorrendo. A regulação, contratualização eoferta de serviços hospitalares, numa lógica de mero cumprimento burocrático, levaram e mantiveram (in) consequentes pulverizações de recursos públicos e, de fato, reforçam os (des) caminhos da implementação do SUS e o quadro que se observa na assistência médico hospitalar no Brasil, com desperdício de recursos eo uso de instrumentos desconexos de regulação, com fortes indícios de captura pelas evidências apresentadas neste artigo.

\section{Colaboradores}

SF Farias foi responsável pela realização da pesquisa, análise dos dados e redação do trabalho. GD Gurgel Júnior participou da revisão eredação do artigo final. AM Costa foi responsável pela orientação e revisão do trabalho. RL Brito e RR Buarque participaram da revisão da literatura. 


\section{Referências}

1. Busse R. Regulation in health care: a basic introduction. In: Conferência: Regulação econômica, concorrência e serviços de interesse geral; 2001; Lisboa: IPE.

2. Castro JD. Regulação em saúde: análise de conceitos fundamentais. Sociologias 2002; 7:122-135.

3. Fiani R. Afinal, a quais interesses serve a regulação? Economia e Sociedade 2004; 13:81-105.

4. Farias LO, M elamed C. Segmentação de mercados da assistência à saúde no Brasil. Cien Saude Colet 2003; 8(2):585-598.

5. M agalhães Júnior H M . Regulação assistencial: a busca de novas ferramentas no SUS para enfrentar 0 desafio de garantir a assistência com equidade. Belo H orizonte: Prefeitura M unicipal; 2002.

6. Santos NR. Encruzilhada nos rumos do SUS: conside rações. [site da Internet] [acessado 2008 mar 07]. Disponível em: http://www.cebes.org.br/anexos/ ENCRUZILHADA\%20D 0\%20SUS_mar\%C3\%A 70\% 2008_2a\%20vers\%C3\%A30.pdf

7. Brasil. Ministério da Saúde. Departamento de Informática do SUS. [acessado 2007 mar 16]. Disponível em: http://w3.datasus.gov.br/datasus/datasus.php

8. Mendes EV. Reflexões sobre a NOAS SUS 01/02. [site da Internet] [acessado 2007 mar 16]. Disponível em: http://www.sms.rio.rj.gov.br/servidor/media/ noas.doc

9. Ribeiro JM. Regulação e contratualização no setor saúde. In: Negri B, Di Giovanni G, organizadores. Brasil: radiografia da saúde. Campinas: Instituto de Economia/Universidade Estadual de Campinas; 2001. p. 409-443.

10. Instituto Brasileiro de Geografia e Estatística. Estatísticas da saúde: assistência médico-sanitária. [site da Internet] [acessado 2007 mar 16]. Disponível em: http://www.ibge.gov.br/home/estatistica/populacao/ condicaodevida/ams/2005/comentarios.pdf

11. Matos CA, Pompeu JC. Onde estão os contratos? Análise da relação entre os prestadores privados de serviços de saúde e o SUS. Cien Saude Colet 2003; 8(2):629-643

12. Bittencourt AS, Camacho LAB, Leal M C. O Sistema de Informação Hospitalar e sua aplicação na saúde coletiva. Cad Saude Publica 2006; 22:19-30.

13. Walshe K. Regulating healthcare: a prescription for improvement? Philadelphia: Open University Press; 2003.

14. Ayres I, Braithwaite J. Responsive Regulation: Transcending the Deregulation Debate. New York: Oxford University Press; 1992.

15. Almeida CM. Os modelos de reforma sanitária dos anos 80: Uma análise crítica. Saude soc. 1996; 5:3-57.
16. Giovanella L. Solidariedade ou competição: políticas e sistema de saúde na Alemanha. Rio de Janeiro: Fiocruz; 1998. p. 139-184.

17. Harrison S, Smith C. Neo-bureaucracy and Public $\mathrm{M}$ anagement: the case of medicine in the $\mathrm{N}$ ational Health Service. Competition \& Change 2003; 7(4):243-254

18. Gurgel Jr. GD, M edeiros KR. Conflito e resistência à mudança no setor saúde: uma análise dos seus determinantes estruturais. In: Ministério da Saúde. Observatório de Recursos Humanos em Saúde no Brasil. Rio de Janeiro: Fiocruz; 2003. p. 37-44.

19. Harrison $S, M$ oran $M$. Resources and rationing: managing supply and demand in health care. In: Albrecht GL, editor. The handbook of social studies in health and medicine. New York: Sage; 2000. p. 493-508.

20. Faveret FP, Oliveira PJ. A universalização excludente: reflexões sobre as tendências do sistema de saúde. Dados 1990; 2:257-283.

21. Barros M E, Piola SF, Vianna SM. Política de Saúde no Brasil: diagnóstico e perspectivas. Brasília: IPEA; 1996. [Texto para discussão no 401]

22. Costa NR. Direito à saúde na Constituição: um primeiro balanço. Cad Saude Publica 1989; 5(1):98-104

23. Gama A. Caracterização da autogestão no processo de regulamentação do setor suplementar [dissertação]. Rio de Janeiro (RJ): Escola Nacional de Saúde Pública, Fundação Oswaldo Cruz; 2003.

24. Brasil. Ministério da Saúde. Secretaria da Atenção à Saúde. Departamento de Regulação, Avaliação e Controle. Coordenação Geral de Regulação e Avaliação da Saúde. Caderno da SAS: Orientações para Contratação de Serviços de Saúde. Brasília: Ministério da Saúde; 2003.

25 Santos FP, M erhy EE. A regulação pública da saúde no Estado brasileiro: uma revisão. Interface (Botucatu) 2006; 10(19):25-41.

26. M endonça CS, Reis AT, Moraes JC, organizadores. A Política de regulação do Brasil. [site da Internet] 2006 [acessado 2008 mai 16] Disponível em: http:// www.bvsms.saúde.gov.br/bvs/ publicacoes/st12.pdf

Artigo apresentado em 13/10/2008

Aprovado em26/02/2009

Versão final apresentada em 26/03/2009 